\title{
Non-absorbable glucids (active hexose correlated compound, inulin and fructooligosaccharides) exert immunomodulatory effects and induce differentiation in several intestinal cell types that are independent of their prebiotic actions
}

\author{
F. Sánchez De Medina ${ }^{1}$, E. Martinez-Plata ${ }^{2}$, I. Romero ${ }^{1}$, M. Ortega ${ }^{2}$, R. Gonzalez ${ }^{1}$, A. Daddaoua ${ }^{2}$, \\ P. Martinez-Moya ${ }^{2}$, A. Zarzuelo ${ }^{1}$, M. D. Suárez ${ }^{2}$ and O. Martínez-Augustin ${ }^{2}$ \\ ${ }^{1}$ Department of Pharmacology, CIBER-EHD, and ${ }^{2}$ Department of Biochemistry and Molecular Biology II, CIBER-EHD, \\ University of Granada, Spain
}

Non-absorbable glucids (NAG) like active hexose correlated compound (AHCC), inulin or fructooligosaccharides (FOS) are known to act as prebiotics, but they could exert different actions independent of their prebiotic activity. Here, we have tested the hypothesis that AHCC, inulin or FOS can regulate cytokine production in monocytes and enterocytes and induce differentiation in enterocytes in a prebiotic independent way.

AHCC is a product prepared from the mycelium of edible Basidiomycete fungi that contains oligosaccharides (74\%), considered the main active ingredient of this product, Of these, nearly $20 \%$ are partially acetylated alpha- 1,4 glucans. To test the influence of AHCC, inulin and FOS on IL-1 $\beta$, IL-8 and TNF production by monocytes and the effect on enterocyte IL-8 production we added them (0.1-5 g/l of culture medium) to THP-1 cells, human peripheral blood monocytes, HT-29 cells and Caco-2 cells cultured to confluency and 1 week after confluency. The effects of lipopolysaccharide (LPS) added to the culture medium was also studied. After the addition of the products, cells were incubated for $24 \mathrm{~h}$ and cytokines were measured in the culture medium by ELISA. The involvement of the MAPK and the NFКB signalling pathways was studied adding different inhibitors (PD98059 for the MAPK ERK1/2, SB203580 for p38 MAPK, SP600125 for c-Jun N-terminal kinase (JNK) and Bay11-7082 for I $\mathrm{KB} \alpha$ ) to the cell culture medium $1 \mathrm{~h}$ before the products. Translocation of p50 and p65 to the nucleus was also studied. Confluent Caco-2 cells were used to study the effect of AHCC on disaccharidase and alkaline phosphatase activities as markers of intestinal differentiation.

Our results show that AHCC increases the production of all the cytokines studied, while inulin and FOS only increase the production of IL-8. The effects of all the products assayed is dependent on both the effect on the MAPK and the NF- $\kappa$ B signalling pathways (Fig. 1). On the other hand, our results indicate that AHCC has no effect in the production of IL-8 either in HT-29 or Caco- 2 cells in basal conditions. Nevertheless in both cell lines it inhibits IL-8 production after LPS stimulation. This effect is independent of the NF- $\kappa B$ signalling pathway since no decrease in IKB phosphorylation of nuclear p50 or p65 levels was observed in HT-29 cells. On the other hand AHCC added to confluent Caco-2 cells led to an increase in the activities of lactase, sucrase and maltase.

The NAG tested stimulates the production of cytokines in monocytes, although AHCC exhibits a broader stimulatory effect. Furthermore, AHCC inhibits enterocyte IL-8 production after LPS stimulation and could induce enterocyte differentiation. In conclusion, our results indicate that NAG exert immunomodulatory and pro differentiation effects that are independent of their prebiotic actions. Therefore, our work describes a new mechanisms of action of NAG.

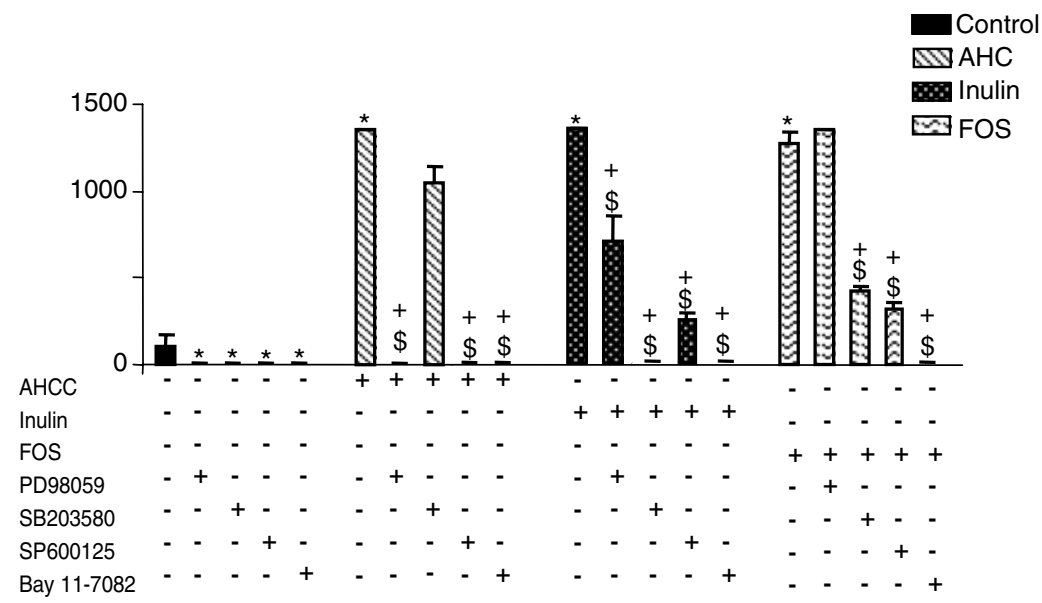

Fig. 1. AHCC, inulin and FOS increase on IL- 8 production through the activation of MAPK and NFKB signalling pathways. * $v$. C; $+\mathrm{C} v$. inhibitor; $\$ v$. AHCC, inulin, FOS. 